\title{
Bim regulation miRrors microRNA $17 \sim 92$ cluster expression in endothelial cells in vivo
}

\author{
XW Meng ${ }^{\star, 1,2}$ and SH Kaufmann*,1,2 \\ Cell Death and Differentiation (2014) 21, 1665-1666; doi:10.1038/cdd.2014.127
}

Sometimes disparate fields come together in interesting ways. Elsewhere in this issue, Koenig et al. ${ }^{1}$ bring together research examining regulation of blood vessel survival in the developing retina and regulation of Bim expression in endothelial cells with intriguing results.

Several previous studies have examined cell death in hyaloid vessels. This transient vascular network provides circulation to the developing eye prior to retinal vascularization but ordinarily regresses during early postnatal development. ${ }^{2}$ Prior studies have shown that hyaloid vessel regression requires Bax and $\mathrm{Bak},{ }^{3}$ suggesting involvement of the intrinsic apoptotic pathway. Moreover, interruption of the Bc/2/11 gene encoding the BH3-only $\mathrm{Bcl}-2$ family member $\mathrm{Bim}$ also diminishes hyaloid vessel regression, ${ }^{4}$ implicating Bim in the process.

A different body of literature has previously investigated various aspects of Bim regulation. As summarized in Figure 1, in healthy cells Bim is bound to and sequestered by dynein light chain $2,{ }^{5}$ which resides on microtubules. Upon phosphorylation by JNK, ${ }^{6}$ however, Bim is released from microtubules and directly binds to Bax or Bak to trigger apoptosis. ${ }^{7,8}$ Apoptosis can also be triggered if Bim expression increases, e.g., as a consequence of Foxo3a- or E2F1-mediated Bc/2/11 transcriptional activation, 9,10 and Bim levels exceed the binding capacity of dynein light chain 2 and other neutralizers, including antiapoptotic Bcl-2 family members. Conversely, cells are protected from apoptosis when Bim expression is downregulated by miR17 92 family microRNAs. ${ }^{11,12}$ In addition, phosphorylation of Bim by RSK2 creates a phosphodegron that is recognized by the E3 ligase $\beta$ TRCP to produce Bim ubiquitylation and degradation, again protecting cells. $^{13}$

In the context of these prior findings, Koenig et al. ${ }^{1}$ asked how Bim is activated to cause physiological vessel loss in the developing retina. Initial experiments not only confirmed that $\mathrm{Bim}^{-1-}$ pups exhibit attenuated postnatal hyaloid vessel regression, but also demonstrated by in situ hybridization that Bim mRNA is upregulated in endothelial cells, pericytes and ocular macrophages of $\mathrm{Bim}^{+/+}$mice as these vessels regress, pointing in the direction of increased gene expression or mRNA stabilization as a contributing factor in this process.
To assess which pathway might be responsible for increased Bim mRNA, Koenig et al. ${ }^{1}$ then examined hyaloid vessels in mice in which all Foxo binding sites in the Bcl2/11 promoter had been mutated or in mice lacking the Foxo3 gene. In both cases, hyaloid vessel regression proceeded normally, suggesting that Foxo-mediated Bcl2/11 transactivation is dispensable for hyaloid vessel regression.

In further experiments, Koenig et al. investigated the role of the miR-17 92 microRNA cluster in Bim-regulated endothelial cell apoptosis. Although miR-17 $~ 92$ cluster miRNAs have been implicated mainly in regulating lymphocyte apoptosis, ${ }^{11,12}$ Koenig et al. ${ }^{1}$ showed that growth factor withdrawal also resulted in reduced miR-17-5P and miR-92-3P miRNA levels in endothelial cells. Further, Bim mRNA levels were elevated in miR-17 $\sim 92^{+/-}$endothelial cells compared to miR-17 $\sim 92^{+1+}$ endothelial cells even under basal conditions, i.e., in the presence of growth factors. When miR$17 \sim 92^{+I-}$ endothelial cells were deprived of growth factors, Bim mRNA levels increased still more. ${ }^{1}$

While these results are consistent with a role for miR$17 \sim 92$ regulation of $\mathrm{Bim}$ in ocular vessels during postnatal development, the present study leaves a number of issues for future study. First, it remains to be shown that hyaloid vessel regression is impaired in $\mathrm{miR}-17 \sim 92^{+/-}$pups. Second, it is unclear whether another factor also contributes to the Bim upregulation during growth factor withdrawal. While the results of Koenig et al. demonstrate that Bim is elevated in miR-17 $\sim 92^{+/-}$endothelial cells under growth factor-replete conditions, the further increase in Bim mRNA in miR$17 \sim 92^{+I}$ cells under starvation conditions suggests an additional level of regulation that might not be completely explained by the data of Koenig et al. Third, given the role of Bim in demise of endothelial cells after treatment with VEGFA-neutralizing antibody, ${ }^{14}$ the role of miR-17 92 cluster RNAs in vessel regression after treatment with bevacizumab for cancer or ranibizumab for wet macular degeneration needs further investigation. Fourth, in view of the recent demonstration that changes in miR-17 92 cluster microRNAs contribute to glucocorticoid-induced Bim upregulation and lymphoid cell death, ${ }^{15}$ one wonders whether changes in miR-17 92 cluster microRNAs play a role in the ability of a wide range of additional anticancer treatments, including histone deacetylase inhibitors, ${ }^{10,16}$ MEK inhibitors ${ }^{17}$ and

\footnotetext{
${ }^{1}$ Division of Oncology Research, Department of Oncology, Mayo Clinic, Rochester, MN 55905, USA and ${ }^{2}$ Department of Molecular Pharmacology and Experimental Therapeutics, Mayo Clinic, Rochester, MN 55905, USA

*Corresponding author: XW Meng or SH Kaufmann, Division of Oncology Research, Department of Oncology, Mayo Clinic, 200 First Street, S.W. Rochester, MN 55905, USA. Tel: +1 507284 8950; Fax: +1 507293 0107; E-mail: Kaufmann.scott@mayo.edu or Meng.xue@ mayo.edu
} 


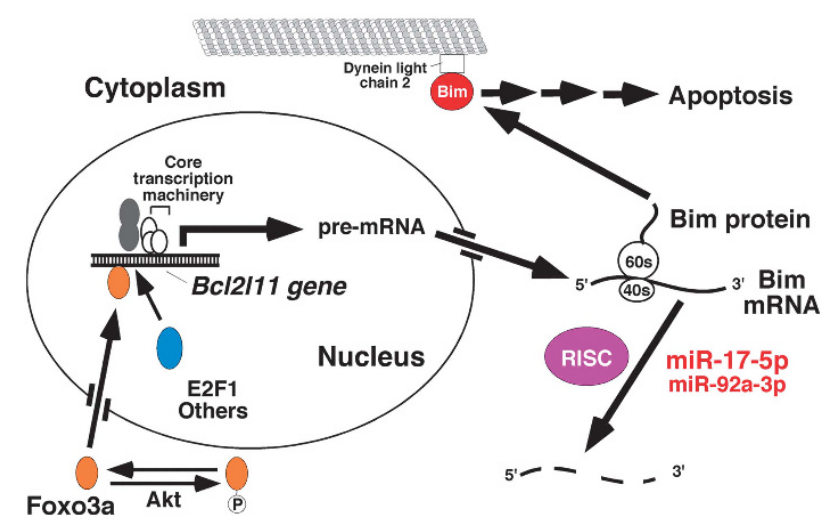

Figure 1 Bim expression is regulated at multiple levels. Transcriptional activation of the Bcl2/11 gene, which encodes Bim message, reflects the activity of Foxo3a, E2F1 and possibly other transcription factors. In addition, levels of Bim mRNA are regulated by microRNAs, including miR-17 $\sim 92$ cluster members miR17-5p and miR-92a-3p, which induce Bim mRNA degradation through the RNAinduced silencing complex (RISC). Once translated, the most abundant isoforms $\mathrm{Bim}_{\mathrm{EL}}$ and $\mathrm{Bim}_{\mathrm{L}}$ are bound and sequestered by dynein light chain 2 in healthy cells ${ }^{5}$ but are released as a consequence of signaling that induces apoptosis. As described elsewhere in this issue, Koenig et al. ${ }^{1}$ have demonstrated that Bim mRNA levels are elevated in endothelial cells during hyaloid vessel regression in the developing eye. Their further studies have shown that this elevation of Bim message does not require Foxo transcription factors, but instead reflects changes in miR-17$5 p$ and, to a smaller extent, miR-92a-3p

tyrosine kinase inhibitors, ${ }^{18}$ to elevate Bim levels and kill cells. Finally, it will be important to further understand what regulates expression of $\mathrm{miR}-17 \sim 92$ cluster microRNAs. Studies reporting that these microRNAs are elevated in and contribute to the pathogenesis of a number of neoplasms, including neuroblastoma, renal cell carcinoma, prostate cancer and various lymphomas, ${ }^{19,20}$ coupled with emerging understanding of their role in suppressing Bim, suggests that this will be a fruitful area of research. Thus, like most good scientific reports, the results of Koenig et al. answer one set of questions only to raise an entirely new set.

\section{Conflict of Interest}

The authors declare no conflict of interest.

Acknowledgements. We thank Deb Strauss for editorial assistance. Preparation of this editorial was supported in part by R01 CA166741.

1. Koenig MN et al. Cell Death Differ 2014 (this issue).

2. Saint-Geniez M, D'Amore PA. Int J Dev Biol 2004; 48: 1045-1058.

3. Hahn P et al. Arch Ophthalmol 2005; 123: 797-802.

4. Wang S, Park S, Fei P, Sorenson CM. Dev Biol 2011; 349: 296-309.

5. Puthalakath H et al. Mol Cell 1999; 3: 287-296.

6. Lei K, Davis RJ. Proc Natl Acad Sci USA 2003; 100: 2432-2437.

7. Dai H et al. J Cell Biol 2011; 194: 39-48.

8. Czabotar PE et al. Cell 2013; 152: 519-531.

9. Stahl $\mathrm{M}$ et al. Immunology 2002; 168: 5024-5031.

10. Zhao Y et al. Proc Natl Acad Sci USA 2005; 102: 16090-16095.

11. Ventura $A$ et al. Cell 2008; 132: 875-886.

12. Xiao C et al. Nat Immunol 2008; 9: 405-414.

13. Dehan E et al. Mol Cell 2009; 33: 109-116.

14. Naik E et al. J Exp Med 2011; 208: 1351-1358.

15. Harada M et al. Leuk Lymphoma 2012; 53: 2041-2050.

16. Lindemann RK et al. Proc Natl Acad Sci USA 2007; 104: 8071-8076.

17. Cragg MS et al. J Clin Invest 2008; 118: 3582-3584.

18. Kuroda J et al. Proc Natl Acad Sci USA 2006 (in press).

19. Lawrie CH. Br J Haematol 2013; 160: 571-581.

20. Hsu T-I et al. Oncogenesis 2014; 3: e99. 